\title{
Correction to: Machine Learning Assisted OSP Approach for Improved QoS Performance on 3D Charge-Trap Based SSDs
}

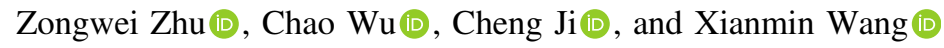

Correction to:

Chapter "Machine Learning Assisted OSP Approach for Improved QoS Performance on 3D Charge-Trap Based SSDs" in: X. Chen et al. (Eds.): Machine Learning for Cyber Security, LNCS 12488, https://doi.org/10.1007/978-3-030-62463-7_9

In the published version the subfigures (g) and (h) in Fig. 7 have been removed.

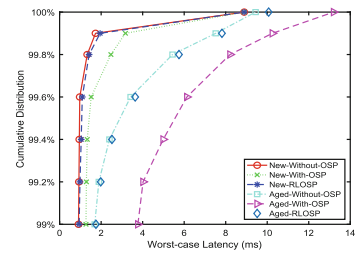

(a) HM_0

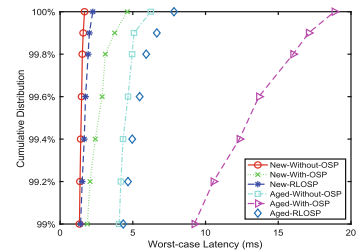

(d) RSRCH_1

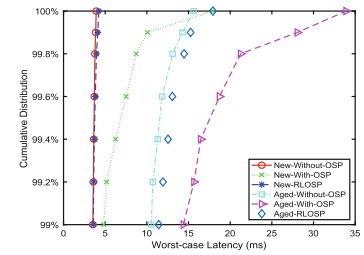

(b) PRN_0

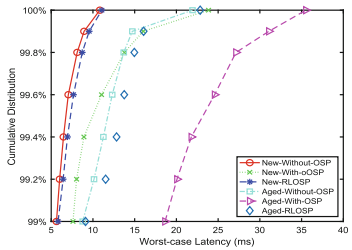

(e) SRC1_2

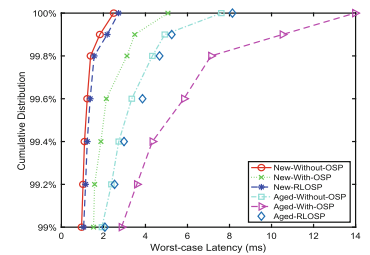

(c) RSRCH_0

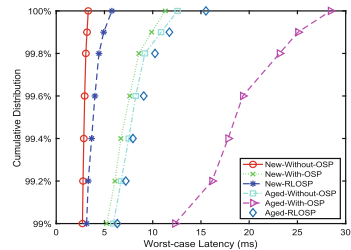

(f) $\mathrm{SRC}_{-} 2$ 